\title{
EFFECT OF 2-DAY VERY LOW-CALORIE DIET ON PERCEIVED STRESS, COGNITIVE FUNCTIONS AND MOOD IN OVERWEIGHT AND OBESE MEN
}

\author{
Rima Solianik, Artūras Sujeta, Agnė Čekanauskaitė, Mantė Januševičiūtė \\ Lithuanian Sports University, Kaunas, Lithuania
}

\begin{abstract}
Background. It is well established that intermittent long-term fasting, during which a very low-calorie diet is allowed 2-days a week, improves weight loss, mood and cognitive functions. However, we are not aware of any previous studies on the effect of a 2-day very low-calorie diet on brain functioning. Thus, the purpose of this study was to determine the effect of 2-day very low-calorie diet on perceived stress, cognitive functions and mood in overweight and obese men.

Methods. Nine young adult men (body mass index (BMI) from 26.7 to $32.1 \mathrm{~kg} / \mathrm{m}^{2}$ ) completed a 2-days very low-calorie diet $(536 \mathrm{kcal})$ and 2-day usual diet program in a randomized order. Perceived stress (subjective stress rating and cardiovascular response), cognitive performance, and mood were evaluated before and after both diets.

Results. A subjective stress rating in relation to the very low-calorie diet condition was $37.4 \pm 18.7$. There were no detectable effects of acute calorie restriction on cardiovascular response (heart rate and blood pressure) and on any aspect of cognitive performance. Meanwhile, sense of vigor increased $(p<.05)$ and negatively correlated with BMI $(r=-.780, p<.05)$.

Conclusions. The two-day very low-calorie diet evoked a mild stress, which did not affect sympathetic nervous system response and cognitive performance in overweight and obese men. Furthermore, very low-calorie diet increased vigor, which was strongly associated with decreased body mass index.
\end{abstract}

Keywords: adiposity, cognitive performance, body mass index, fasting, heart rate.

\section{INTRODUCTION}

A ccording to the World Health Organization (WHO, 2016), the obesity epidemic has more than doubled since 1980. In 2014, approximately $13 \%$ of the world's adult population were obese. Over 1.9 billion adults $(18+$ years $)$ were overweight, with more than 600 million being obese (WHO, 2016).

With the increasing obesity epidemic, the need for effective and safe approaches to weight loss occurs. One of the examples is widely known 5:2 intermittent fasting, in the case of which a very lowcalorie diet is allowed 2 days a week and "normal" eating is resumed on non-diet days (Johnstone, 2015). It is well known that long-term fasting improves health, reduces risks of many chronic diseases, cognitive functions and mood (Longo \&
Mattson, 2014. However, we are not aware of any previous studies on the effect of a 2-day very lowcalorie diet on cognitive functions and mood in overweight and obese men.

It was observed that low-calorie dieting increases the cortisol level and perceived stress (Tomiyama et al., 2010); therefore, a critical transition period of 3-6 weeks during which brain adapts to intermittent fasting is needed (Longo \& Mattson, 2014). Thus, it can be expected that acute calorie restriction during first two days will lead to stress which will adversely affect cognitive functions and mood. Therefore, the aim of this study was to estimate the effect of 2-day very lowcalorie diet on perceived stress, cognitive functions and mood in overweight and obese men. 


\section{METHODS}

Research participants. Ten men were assessed for eligibility. The inclusion criteria were as follows: young (aged 18-35 years), overweight or mildly obese (body mass index (BMI) $25-34.9 \mathrm{~kg} /$ $\mathrm{m}^{2}$ ) men. Participants were excluded if they were in a weight-loss program, smokers, regularly used medications, had a history of acute or chronic diseases, eating disorders, and those who had previously suffered a head trauma. In total, 9 men aged $28.7 \pm 5.1$ years met the inclusion criteria and agreed to participate in this study. Their physical characteristics are presented in Table 1. Written informed consent was obtained from all participants. All procedures were conducted according to the principles of the Declaration of Helsinki.

Procedures. Four days before the experiment, the participants were familiarized with the laboratory setting and with the experimental procedures used for cognitive function evaluation. During familiarization, the participants were instructed to refrain from ingesting alcoholic beverages, caffeine, and from intense physical and mental work for at least $48 \mathrm{~h}$ before the experimental measurements.

During the second and third visits, participants underwent either an experimental 2-day very low calorie diet (536 kcal/day) or a control usual diet in a randomized order, at least 2 weeks apart. Both experiments began at 8:00 a.m. The participants arrived at the laboratory after overnight fasting (8-12h) to complete baseline measurements. On arrival at the laboratory, anthropometric measurements were performed. Then the participants were asked to rest in a sitting position for $10 \mathrm{~min}$ in a quiet room. Subsequently, control measurements of resting heart rate (HR), blood pressure (BP) and glucose level were performed, and the participants rated their mood. Then, the participants took their seats at a table in a welllit room, and cognitive functions were evaluated. Afterwards, the participants rested 1 day before starting the 2-day very low-calorie diet or 2-day usual diet. During usual diet participants were requested to make no changes in their daily diet. Meanwhile, during the very low-calorie diet they were given two sachets per day of instant oatmeal porridge of strawberries taste ACTIVUS (Kauno grūdai, Lithuania) and unlimited amounts of water. Each oatmeal porridge contained 268 kilocalories:
$48 \mathrm{~g}$ carbohydrates, $9.7 \mathrm{~g}$ sugars, $7.6 \mathrm{~g}$ protein, $6.1 \mathrm{~g}$ fat, $5.4 \mathrm{~g}$ dietary fibers, $1.6 \mathrm{~g}$ saturated fatty acids, and $0.57 \mathrm{~g}$ salt. Both diets were followed by the performance of experimental measurements in the same order as described before dieting. In addition, the participants rated their stress level in relation to the diet.

Measurements. Anthropometric evaluation. The participant's weight (TBF-300 body composition scale, Tanita, UK) and height (Leicester Height Meter, Invicta Plastics, UK) were estimated while the subjects were wearing only underwear and barefoot. BMI was calculated as body weight divided by squared height.

Evaluation of stress level. Subjective stress rating in relation to calorie restriction was assessed on a visual analog scale ranging from 0 ("no stress") to 100 ("the highest stress imaginable") on a $10 \mathrm{~cm}$ long horizontal line.

Resting HR was recorded while seated using a HR monitor (S-625X, Polar Electro, Finland) affixed to the chest. In addition, indirect arterial BP measurement was taken from the upper arm using a sphygmomanometer (Sanaphon, Germany) and a standard-size arm cuff.

Evaluation of glucose level. The blood glucose level was determined in capillary blood samples using a validated CardioCheck PA analyzer (Polymer Technology Systems Inc, USA) via finger-prick test (Panz, Raal, Paiker, \& Immelman, 2005).

Evaluation of mood state. The Brunel Mood Scale was used to assess current mood state (Terry, Lane, \& Fogarty, 2003). This questionnaire contains of 24 items divided into six subscales: anger, confusion, depression, fatigue, tension, and vigor. The items are answered on a five-point scale ranging from 0 ("not at all") to 4 ("extremely"), and each subscale, with four relevant items, are summed to produce a raw score in the range of 0 to 16 .

Evaluation of cognitive function. The computerized automated neuropsychological assessment metric Version 4 (ANAM-4; Vista Life Sciences, USA) was used to assess simple motor reaction time (Simple Reaction Time Task) (Woodhouse et al., 2013; Reeves, Winter, Bleiberg, \& Kane, 2007), mental flexibility (TwoChoice Reaction Time Task) (Solianik, Sujeta, Terentjeviene, \& Skurvydas, 2016; Reeves, Winter, Bleiberg, \& Kane, 2007), inhibitory control (Go/ No-Go Task) (Diamond, 2013), verbal working memory (Memory Search Task) (Reeves, Winter, 
Bleiberg, \& Kane, 2007), and spatial processing and visuospatial working memory (Matching to Sample Task) (Vincent, Roebuck-Spencer, Gilliland, \& Schlegel, 2012). During Simple Reaction Time Task, simple stimulus "*” was presented on the screen and the participant was instructed to respond as quickly as possible by pressing the designated button each time the stimuli was presented. During Two-Choice Reaction Time Task, one of two stimuli was presented on the screen ("*") or "o") and the participant was instructed to respond as quickly as possible by pressing the designated button for each stimulus. During Go/No-Go Task, the participant was instructed to respond as quickly as possible to an " $\mathrm{x}$ " on the screen by pressing the designated button each time the stimulus appeared; when an "o" appeared, the participant was required to withhold his response. During Memory Search Task, a string of six letters was presented for memorization. The participant was instructed to press the designated button once he had memorized the letter string; then, it disappeared from view and individual letters were presented one at a time. The participant was instructed to indicate as quickly as possible if the letter belongs to the memorized set and press designated button for memory set letters and another designated button for letters not included in the memory set. During Matching to Sample Task, a pattern produced by eight shaded cells in a $4 \times 4$ sample grid was presented for 2 $\mathrm{s}$ and then disappeared. After $5 \mathrm{~s}$, two patterns are presented side-by-side and the participant was instructed to indicate as quickly as possible by pressing the designated button for the grid that matches the previously shown sample.

Statistical analyses. Data are reported as means \pm standard deviations. The data were tested for normal distribution using the KolmogorovSmirnov test, and all data were found to be normally distributed. To assess the effects of diets on variables measured (i.e. anthropometric characteristics, HR, BP, glucose level, mood and cognitive functions), and compare data between the usual diet results with those of the very lowcalorie diet, the parametric paired-sample t-tests were performed. The magnitudes of the 2-day very low-calorie diet evoked changes were estimated by Cohen's d effect sizes. Pearson correlation coefficients (r) were used to identify relationships between changes ( $\Delta=$ value before the very lowcalorie diet - value after this type of diet) in mood and anthropometric measurements. The level of significance was set at $p<.05$. If a significant time effect was found, the statistical power (SP) was calculated. All statistical analyses were performed using SPSS (version 21.0; IBM Corp., USA).

\section{RESULTS}

Effect of a very low-calorie diet on anthropometric measurements. The anthropometric characteristics of the participants in this study are presented in Table 1 . The very low-calorie diet decreased weight $(p=.001, \mathrm{SP}=99.3 \%, \mathrm{ES}=0.156)$ and BMI $(p<.001, \mathrm{SP}=100.0 \%, \mathrm{ES}=0.248)$. As expected, the usual diet did not induce any changes in anthropometric characteristics. Lower weight $(p=.003)$ and lower BMI $(p=.002)$ were observed after very low-calorie diet compared with the usual diet.

Effect of a very low-calorie diet on stressrelated measurements. A subjective stress rating in relation to the very low-calorie diet condition was $37.4 \pm 18.7$. As presented in Table 2, the very low-calorie diet had no effect on HR and BP. Comparison of the diets showed no significant differences in stress-related markers.

Effect of a very low-calorie diet on glucose level. As presented in the Figure, the very lowcalorie diet had no effect on glucose level. Comparison of the diets showed no significant differences in glucose level.

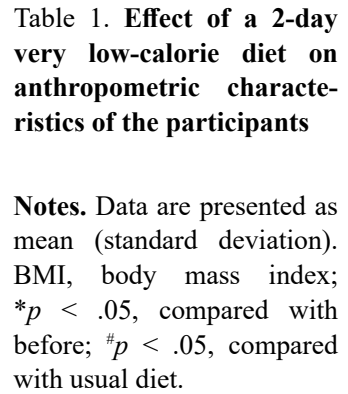

\begin{tabular}{|l|c|c|c|c|}
\hline \multirow{2}{*}{} & \multicolumn{2}{|c|}{ Usual diet } & \multicolumn{2}{c|}{ Very low-calorie diet } \\
\cline { 2 - 5 } & Before & After & Before & After \\
\hline Mass $(\mathrm{kg})$ & $98.4(12.5)$ & $98.1(12.4)$ & $97.2(12.0)$ & $95.3(11.4)^{* \#}$ \\
\hline BMI $\left(\mathrm{kg} / \mathrm{m}^{2)}\right.$ & $28.3(2.3)$ & $28.2(2.2)$ & $28.0(2.2)$ & $27.4(2.1)^{* \#}$ \\
\hline
\end{tabular}


Effect of a very low-calorie diet on mood. The mood state of the participants is presented in Table 3. The very low-calorie diet significantly increased vigor $(p=.041, \mathrm{SP}=92.6 \%, \mathrm{ES}=0.931)$, whereas other mood states were not affected. The vigor negatively correlated with BMI $(r=-0.780, p=.023)$ and tended to negatively correlate with the body weight $(r=$ $-0.685, p=.061)$. As expected, the usual diet did not induce any changes in the mood of the participants. Comparison of the diets showed greater baseline vigor $(p=.007)$ before the usual diet.

Effect of a very low-calorie diet on cognitive performance. As presented in Table 4, the very lowcalorie diet had no effect on cognitive performance. Comparison of the diets showed no significant differences in cognitive performance.

\begin{tabular}{|l|c|c|c|c|}
\hline \multirow{2}{*}{} & \multicolumn{2}{|c|}{ Usual diet } & \multicolumn{2}{c|}{ Very low-calorie diet } \\
\cline { 2 - 5 } & Before & After & Before & After \\
\hline Heart rate (bpm) & $64.0(4.3)$ & $64.9(4.9)$ & $71.0(9.5)$ & $65.7(7.6)$ \\
\hline Systolic BP (mmHg) & $115.7(5.6)$ & $112.9(3.6)$ & $117.9(7.0)$ & $113.6(6.9)$ \\
\hline Diastolic BP (mmHg) & $71.4(5.8)$ & $70.7(5.6)$ & $72.1(3.6)$ & $69.3(4.2)$ \\
\hline
\end{tabular}

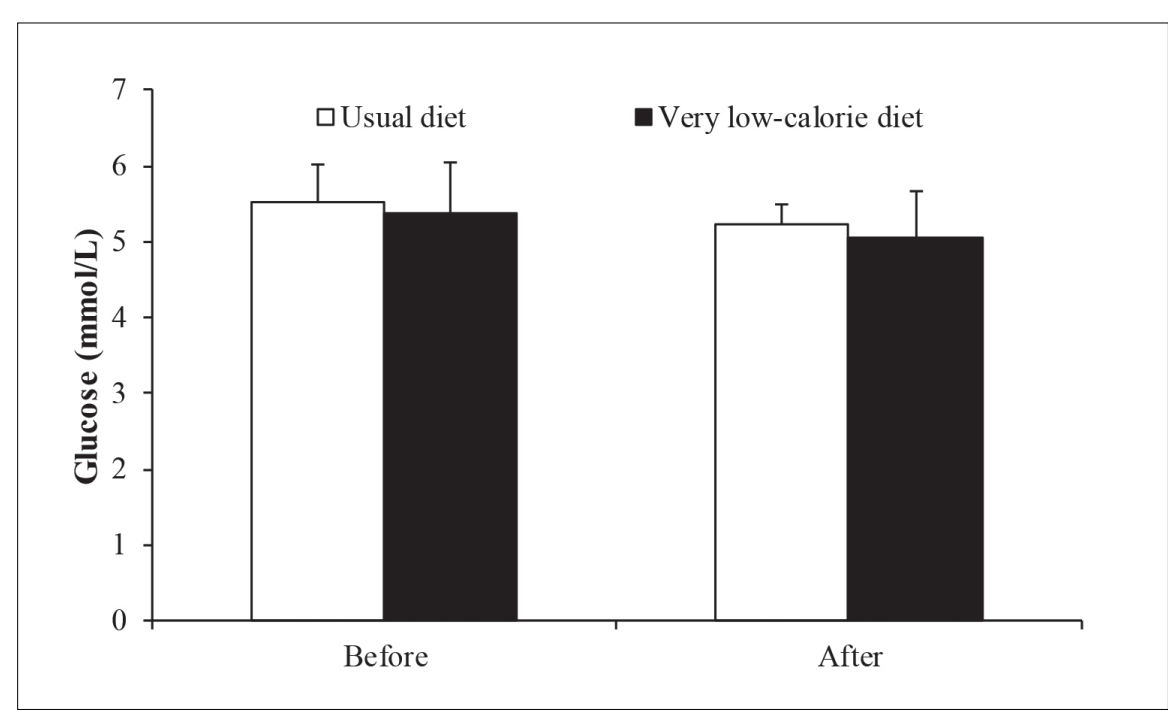

\begin{tabular}{|l|c|c|c|c|}
\hline \multirow{2}{*}{} & \multicolumn{2}{|c|}{ Usual diet } & \multicolumn{2}{c|}{ Very low-calorie diet } \\
\cline { 2 - 5 } & Before & After & Before & After \\
\hline Anger & $0.3(0.2)$ & $0.6(0.9)$ & $1.4(2.0)$ & $1.4(1.4)$ \\
\hline Confusion & $1.9(2.4)$ & $0.6(0.7)$ & $1.9(2.8)$ & $0.7(1.2)$ \\
\hline Depression & $1.4(3.1)$ & $0.1(0.3)$ & $2.3(3.1)$ & $1.4(1.7)$ \\
\hline Fatigue & $3.6(2.1)$ & $4.6(3.3)$ & $5.9(3.9)$ & $3.7(2.9)$ \\
\hline Tension & $2.1(1.8)$ & $1.7(1.3)$ & $1.9(1.5)$ & $2.1(2.2)$ \\
\hline Vigor & $10.2(1.9)$ & $9.0(1.1)$ & $6.3(3.2)^{\#}$ & $9.4(3.5)^{*}$ \\
\hline
\end{tabular}

Table 3. Effect of a 2-day very lowcalorie diet on moo

Notes. Data are presented as mean (standard deviation). $* p<.05$, compared with before; ${ }^{\#} p<.05$, compared with usual diet.

Table 2. Effect of a 2-day very lowcalorie diet on heart rate and blood pressure

Figure. Effect of a 2-day very lowcalorie diet on glucose levels

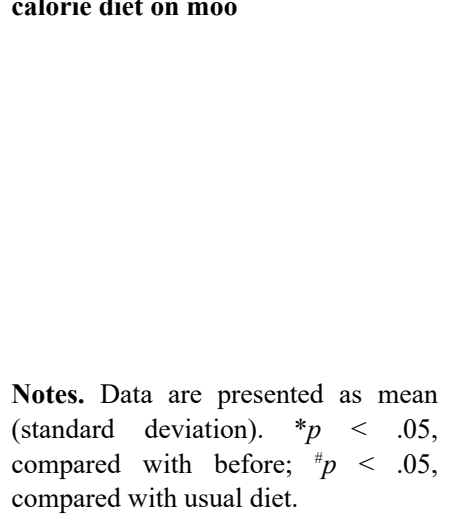


Table 4. Effect of a 2-day very low-calorie diet on cognitive performance

\begin{tabular}{|c|c|c|c|c|}
\hline & \multicolumn{2}{|c|}{ Usual diet } & \multicolumn{2}{|c|}{ Very low-calorie diet } \\
\hline & Before & After & Before & After \\
\hline \multicolumn{5}{|c|}{ Simple Reaction Time Task } \\
\hline Reaction time (ms) & $291.7(13.5)$ & $286.3(11.1)$ & $296.2(23.9)$ & $281.6(24.8)$ \\
\hline \multicolumn{5}{|c|}{ Two-Choice Reaction Time Task } \\
\hline $\begin{array}{l}\text { Reaction time (ms) } \\
\text { Accuracy }(\%)\end{array}$ & $\begin{array}{c}453.4(37.7) \\
93.7(6.8) \\
\end{array}$ & $\begin{array}{c}448.3(37.5) \\
95.5(2.7)\end{array}$ & $\begin{array}{c}464.0(79.7) \\
94.6(3.1)\end{array}$ & $\begin{array}{c}444.9(46.2) \\
94.0(4.0)\end{array}$ \\
\hline \multicolumn{5}{|c|}{ Go/No-Go Task } \\
\hline $\begin{array}{l}\text { Reaction time (ms) } \\
\text { Accuracy }(\%)\end{array}$ & $\begin{array}{c}335.2(18.3) \\
95.7(1.9) \\
\end{array}$ & $\begin{array}{c}335.7(20.1) \\
95.8(2.1)\end{array}$ & $\begin{array}{c}333.3(9.5) \\
95.7(2.8) \\
\end{array}$ & $\begin{array}{c}331.3(10.7) \\
95.5(2.0)\end{array}$ \\
\hline \multicolumn{5}{|c|}{ Memory Search Task } \\
\hline $\begin{array}{l}\text { Reaction time (ms) } \\
\text { Accuracy }(\%)\end{array}$ & $\begin{array}{c}1030.9(183.9) \\
88.9(8.3) \\
\end{array}$ & $\begin{array}{c}903.4(124.8) \\
96.3(2.7) \\
\end{array}$ & $\begin{array}{c}849.0(129.4) \\
92.8(11.7)\end{array}$ & $\begin{array}{c}944.4(134.7) \\
93.8(4 / 2) \\
\end{array}$ \\
\hline \multicolumn{5}{|c|}{ Matching to Sample Task } \\
\hline $\begin{array}{l}\text { Reaction time (ms) } \\
\text { Accuracy }(\%)\end{array}$ & $\begin{array}{c}1572.1(363.8) \\
95.6(4.2)\end{array}$ & $\begin{array}{c}1570.0(383.9) \\
95.5(4.1)\end{array}$ & $\begin{array}{c}1527.3(308.4) \\
95.1(4.6)\end{array}$ & $\begin{array}{c}1652.5(476.9) \\
98.8(2.3)\end{array}$ \\
\hline
\end{tabular}

Note. Data are presented as mean (standard deviation).

\section{DISCUSSION}

The aim of the present study was to determine the effect of 2-day very low-calorie diet on perceived stress, cognitive functions and mood in overweight and obese men. The participants indicated that they sensed mild stress with no effect on sympathetic nervous system markers (HR and BP) and cognitive performance. The 2-day very low-calorie diet improved mood, specifically this improvement was observed in sensing higher vigor, which was associated with decreased BMI.

The very low-calorie diet evoked a mild stress, which was not severe enough to affect physiological stress markers in overweight and obese men. In contrast to complete fasting (Andersson, Wallin, Hedner, Ahlberg, \& Andersson, 1988; Solianik, Sujeta, Terentjeviene, \& Skurvydas, 2016), the 2-day very low-calorie diet did not affect HR and BP responses. Under conditions of complete fasting, ketones are produced in response to a low glucose level (Cunnane, et al., 2011) and may affect the sympathetic nervous system response
(Kimura et al., 2011). It is worth mentioning that under conditions of this study, in contrast to complete (Solianik, Sujeta, Terentjevienè, \& Skurvydas, 2016) or near complete calorie restriction (Lieberman et al., 2008), in the current study glucose level was not changed in overweight and obese men.

In contrast to our expectations, the very lowcalorie diet did not affect cognitive performance in overweight and obese men. It is well established that glucose level (Mergenthaler, Lindauer, Dienel, \& Meisel, 2013) and perceived stress (Sandi, 2013) play an important role in brain functioning. However, as previously mentioned, the glucose level was not affected, and only a mild stress not affecting any sympathetic response was induced.

Contrary to our expectations, we observed that the very low calorie-diet increased the sense of vigor in overweight and obese men. Lieberman et al. (2008) reported that mood was not affected in healthy humans by a 2-day near complete (313 
kcal) calorie restriction, meanwhile a complete calorie restriction deteriorated the mood (Solianik, Sujeta, Terentjevienè, \& Skurvydas, 2016; Uher, Treasure, Heining, Brammer, \& Campbell, 2006). Interestingly, a strong negative relationship was observed between BMI and vigor, and a tendency of moderate negative relationship between weight and vigor was observed as well. It is well established that weight loss has significant effect on positive changes in mood, which is related with active participation in a weight loss program (Wing, Epstein, Marcus, \& Kupfer, 1984). Lieberman et al. (2008) suggested that the double-blind, placebocontrolled procedures can prevent subjective expectations and changes in mood. It might be suggested that the expectations about very lowcalorie diet increased the sense of vigor; thus, in future the double-blind and placebo-controlled procedures should be carried out.

It is important to note that differences between men and women may exist (Solianik, Sujeta,
Terentjevienè, \& Skurvydas, 2016), thus, our results can only be generalized to men. It remains to be established whether the conclusions of this study also pertain to overweight and obese women.

\section{CONCLUSIONS}

To our knowledge, this is the first study to examine the effect of a 2-day very low-calorie diet on perceived stress, cognitive functions and mood in overweight and obese men. Our study showed that a short-term very low-calorie diet resulted in a mild stress, which did not affect sympathetic nervous system response and cognitive response. Furthermore, the increased vigor occurring alongside decreased body mass index was established.

Competing interests. The authors declare that they have no competing interests.

Acknowledgements. This work was supported by the AB Kauno grūdai.

\section{REFERENCES}

Andersson, B., Wallin, G., Hedner, T., Ahlberg, A.-C., \& Andersson, O. (1988). Acute effects of short-term fasting on blood pressure, circulating noradrenaline and efferent sympathetic nerve activity. Acta Medica Scandinavica, 223(6), 485-490. doi:10.1111/j.0954-6820.1988. tb17685.x

Cunnane, S., Nugent, S., Roy, M., Courchesne-Loyer, A., Croteau, E., Tremblay, S., . . . Rapoport, S. (2011). Brain fuel metabolism, aging and Alzheimers disease. Nutrition, 27(1), 3-20. doi:10.1016/j.nut.2010.07.021

Diamond, A. (2013). Executive Functions. Annual Review of Psychology, 64, 135-168. doi:10.1146/ annurev-psych-113011-143750

Johnstone, A. (2015). Fasting for weight loss: An effective strategy or latest dieting trend? International Journal of Obesity, 39(5), 727-733. doi:10.1038/ ijo.2014.214

Kimura, I., Inoue, D., Maeda, T., Hara, T., Ichimura, A., Miyauchi, S., . . Tsujimoto, G. (2011). Short-chain fatty acids and ketones directly regulate sympathetic nervous system via G protein-coupled receptor 41 (GPR41). Proceedings of the National Academy of Sciences of the United States of America, 108(19), 8030-8035. doi:10.1073/pnas.1016088108

Lieberman, H., Caruso, C., Niro, P., Adam, G., Kellogg, M., Nindl, B., \& Kramer, F. (2008). A double-blind, placebo-controlled test of 2D of calorie deprivation: Effects on cognition, activity, sleep, and interstitial glucose concentrations. The American Journal of Clinical Nutrition, 88(3), 667-676.
Longo, V., \& Mattson, M. (2014). Fasting: Molecular mechanisms and clinical applications. Cell Metabolism, 19(2), 181-192. doi:10.1016/j.cmet.2013.12.008

Mergenthaler, P., Lindauer, U., Dienel, G., \& Meisel, A. (2013). Sugar for the brain: The role of glucose in physiological and pathological brain function. Trends in Neurosciences, 36(10), 587-597. doi:10.1016/j. tins.2013.07.001

Panz, V., Raal, F., Paiker, J., \& Immelman, R. M. (2005). Performance of the CardioChek PA and Cholestech LDX point-of-care analysers compared to clinical diagnostic laboratory methods for the measurement of lipids. Cardiovascular Journal of Africa, 16(2), 112-111.

Reeves, D., Winter, K., Bleiberg, J., \& Kane, R. (2007). ANAM $®$ Genogram: Historical perspectives, description, and current endeavors. Archives of Clinical Neuropsychology, 22(Suppl. 1), 15-37. doi:10.1016/j. acn.2006.10.013

Sandi, C. (2013). Stress and cognition. Wiley Interdisciplinary Reviews: Cognitive Science, 4(3), 245-261. doi:10.1002/wcs.1222

Solianik, R., Sujeta, A., Terentjevienè, A., \& Skurvydas, A. (2016). Effect of $48 \mathrm{~h}$ fasting on autonomic function, brain activity, cognition, and mood in amateur weight lifters. BioMed Research International, 1503956. doi:10.1155/2016/1503956

Terry, P., Lane, A., \& Fogarty, G. (2003). Construct validity of the Profile of Mood States - Adolescents for use with adults. Psychology of Sport and Exercise, 4(2), 125-139. doi:0.1016/S1469-0292(01)00035-8 
Tomiyama, A., Mann, T., Vinas, D., Hunger, J., DeJager, J., \& Taylor, S. (2010). Low calorie dieting increases cortisol. Psychosomatic Medicine, 72(4), 357364. doi:10.1097/PSY.0b013e3181d9523c

Uher, R., Treasure, J., Heining, M., Brammer, M., \& Campbell, I. (2006). Cerebral processing of food-related stimuli: Effects of fasting and gender. Behavioural Brain Research, 169(1), 111-119. doi:10.1016/j. bbr.2005.12.008

Vincent, A., Roebuck-Spencer, T., Gilliland, K., \& Schlegel, R. (2012). Automated Neuropsychological Assessment Metrics (v4) Traumatic Brain Injury Battery: Military normative data. Military Medicine, 177(3), 256-269. doi:10.7205/MILMED-D-11-00289
Wing, R., Epstein, L., Marcus, M., \& Kupfer, D. (1984). Mood changes in behavioral weight loss programs. Journal of Psychosomatic Research, 28(3), 189-196. doi:10.1016/0022-3999(84)90019-9

Woodhouse, J., Heyanka, D., Scott, J., Vincent, A., Roebuck-Spencer, T., Domboski-Davidson, K., . . . Adams, R. (2013). Efficacy of the ANAM General Neuropsychological Screening Battery (ANAM GNS) for detecting neurocognitive impairment in a mixed clinical sample. Clinical Neuropsychologist, 27(3), 376385. doi:10.1080/13854046.2012.762427

World Health Organization. (2016). Obesity and overweight fact sheet. Retrieved from http://www.who. int/mediacentre/factsheets/fs311/en/
Corresponding author Rima Solianik Institute of Sports Science and Innovations Lithuanian Sports University

Sporto str. 6, Kaunas LT-44221

Lithuania

Email rima.solianik@1su.1t 